\title{
Optimización de la Hidrólisis Enzimática de Proteínas de Plasma Bovino
}

\author{
Omar A Figueroa ${ }^{(1)}$, José E Zapata ${ }^{(2)^{*}}$ y Claudia P. Sánchez ${ }^{(2)}$ \\ (1) Universidad de la Guajira, Cra 6 \# 24-103 Valledupar Cesar (e-mail: omfimo22@gmail.com) \\ (2) Facultad de Ciencias Farmacéuticas y Alimentarias, Universidad de Antioquia. Medellín, Colombia. \\ Calle 67 №53-108 (e-mail: jedgar_4@yahoo.es; cpatricia.sanchez@udea.edu.co)
}

${ }^{*}$ Autor a quien debe ser dirigida la correspondencia

Recibido Sep. 16, 2015; Aceptado Nov. 17, 2015; Versión final Dic. 24, 2015, Publicado Abr. 2016

\section{Resumen}

Se optimizó la reacción de hidrólisis enzimática de proteínas de sangre bovina en un reactor en lote, empleando la metodología de superficie de respuesta y la estrategia de algoritmos genéticos, como herramientas de optimización. Además, se analizó el efecto de las concentraciones de sustrato sobre la velocidad de reacción por medio de un modelo matemático en el que se asume una cinética de inhibición por sustrato. Para la hidrólisis se utilizó la enzima Alcalase ${ }^{\circledR} 2.4 \mathrm{~L}$. Se analizaron los factores pH, temperatura y relación enzima-sustrato, con el objetivo de alcanzar el máximo grado de hidrólisis en dos horas de reacción, empleando el método de valoración del protón liberado. El efecto de los factores experimentales sobre el máximo grado de hidrólisis se ajustó satisfactoriamente a un modelo polinomial $\left(\mathrm{R}^{2}=0.93\right)$. Las condiciones óptimas para la reacción fueron: temperaturas de $60.4^{\circ} \mathrm{C}, \mathrm{pH}$ de 9 y relación enzima-sustrato de $10 \%(w / w)$, para alcanzar un grado de hidrólisis máximo de $23 \%$.

Palabras clave: grado de hidrolisis; optimización; cinética enzimática; hidrólisis proteica

\section{Optimization of Enzymatic Hydrolysis of Proteins Bovine Plasma}

\begin{abstract}
The enzymatic hydrolysis reaction of bovine blood proteins in a batch reactor using the response surface methodology and Genetic Algorithms as optimization tools was studied. Furthermore, the effect of substrate concentration on the reaction rate using a mathematical model, which assumes substrate inhibition, was analyzed. The Alcalase $\AA 2.4 \mathrm{~L}$ is used for the hydrolysis. The $\mathrm{pH}$, temperature and the enzyme-substrate ratio were analyzed in order to maximize the degree of hydrolysis for two hours of reaction, using the method of the assessment of proton release. The effect of experimental factors on the degree of hydrolysis was satisfactorily adjusted to a polynomial model with $R^{2}=0.93$. The optimal reaction conditions were temperature $60.4{ }^{\circ} \mathrm{C}, \mathrm{pH} 9$ and enzyme-substrate of $10 \%(\mathrm{w} / \mathrm{w})$, to achieve maximum degree of hydrolysis of $23 \%$.
\end{abstract}

Keywords: degree of hydrolysis; optimization; enzymatic kinetic; protein hydrolysis 


\section{INTRODUCCIÓN}

Los hidrolizados de proteínas han sido utilizados en muchos procesos alimentarios gracias a sus propiedades funcionales, como mayor capacidad de agitación, dispersión y elevada solubilidad (Benítez et al., 2008). Del mismo modo, las proteínas y péptidos procedentes de alimentos están siendo ampliamente usados para mejorar funciones biológicas (Möller et al., 2008), pues los péptidos obtenidos por hidrólisis, son capaces de ejercer efectos biológicos específicos (Di Bernardini et al., 2010). Para el caso de los hidrolizados de plasma sanguíneo de cerdo y bovino, se ha reportado capacidad inhibidora de la enzima convertidora de la Angiotensina (ACE), así como su actividad antigenotóxica (reducción de daños en el ADN) (Hyun y Park, 2002) y antioxidante (Liu et al., 2010). Al tiempo que han sido aislados péptidos de hemoglobina bovina con propiedades antimicrobianas (Nedjar-Arroume et al., 2006).

La búsqueda de hidrolizados con propiedades deseables a partir de proteínas, hace necesario desarrollar estudios orientados a encontrar la enzima proteolítica adecuada para cada sustrato. A este respecto la Alcalasa $^{\circledR} 2,4 \mathrm{~L}$ ha sido empleada con mucho éxito. Además de esto es importante reconocer los factores con efectos importantes sobre la hidrólisis, tales como la Temperatura, el pH y la relación enzima sustrato. Debido al hecho de que la mayoría de los problemas en biotecnología son problemas multidimensionales de búsqueda con una gran cantidad de óptimos locales, una estrategia común para el estudio de las condiciones de trabajo de las reacciones de hidrolisis, es desarrollar modelamiento empírico con base en la estadística. Estos modelos suelen ser de gran valor para el análisis de los sistemas complejos. La metodología de superficie de respuesta (RSM) se ha convertido en un método bastante popular para la optimización de variados procesos alimentarios (Qi et al; 2009), y en la actualidad se ha usado con éxito en la hidrólisis de procesos enzimáticos con diferentes sustratos y proteasas (Eslahi et al., 2013; Seo, 2015), sistemas que suelen ser complejos, debido a los variados efectos no lineales que se pueden presentar.

Debido al potencial de aplicaciones del plasma y otras proteínas de la sangre en el área de los alimentos y fármacos, actualmente existe gran interés por modelar el comportamiento de sistemas que involucran reacciones de hidrólisis enzimática, con el fin de dimensionar equipos industriales, pronosticar comportamientos dinámicos, controlar tiempos de proceso y otras variables cinéticas (Arantes, 2008). Es importante resaltar que la optimización de estos procesos depende del conocimiento del comportamiento cinético. En este sentido, un modelo muy simple es insuficiente para representar un proceso y uno muy complejo, se hace poco práctico (Márquez y Vázquez, 1999). Modelos cinéticos para sistemas en lote, que explican la velocidad de hidrólisis de caseínas (Rubio et al., 1993), lactoalbúminas (González-Tello et al., 1994), hemoglobina bovina (Márquez y Vázquez, 1999) y albuminas (Shi et al., 2005), han presentado buenos ajustes, generando información básica para la optimización de los procesos. Fenómenos de desactivación enzimática han sido establecidos experimentalmente, en el análisis de la cinética de hidrólisis enzimática de proteínas del plasma, basado en la ecuación de Michaelis-Menten (M-M) (Figueroa et al, 2012).

En la última década, además de la utilización de técnicas convencionales de optimización basadas en gradiente, se han tratado de establecer nuevos enfoques para la optimización experimental (Patel, 2014), pues técnicas como la MSR están orientadas a encontrar óptimos locales. La optimización global de una función requiere una búsqueda a través de toda la configuración espacial. Con aplicación en problemas no determinísticos de difícil abordaje. Para resolver estos problemas se han propuesto métodos como los AG (Liu et al., 2008). Estos son algoritmos basados en los principios de la evolución por selección natural, es decir, la supervivencia del más apto, que se han establecido como una búsqueda de gran alcance y técnicas de optimización para resolver problemas con funciones objetivo que no son continuas 0 diferenciables (Muffler et al., 2009). Los algoritmos genéticos se han aplicado exitosamente en problemas de optimización que involucran procesos biológicos (Sarkar y Modak., 2003), en donde la finalidad principal es establecer condiciones de procesos que mejoren la productividad de los mismos.

En este artículo, se presenta una aplicación para resolver un problema de optimización de un modelo polinomial obtenido por regresión, luego del análisis experimental para un sistema de reacción de hidrolisis enzimática de plasma entero bovino y una enzima comercial (Alcalasa ${ }^{\circledR} 2,4 \mathrm{~L}$ ), donde tres factores experimentales: $\mathrm{pH}$, temperatura y relación enzima-sustrato $(\mathrm{E} / \mathrm{S})$, se analizaron en pro de maximizar el grado de hidrólisis $(\mathrm{GH})$ en dos horas de reacción, empleando la técnica convencional de MSR y comparándola con una rutina de optimización con AG. Además, se analiza la influencia de la concentración de sustrato sobre la reacción de hidrólisis enzimática de este sustrato con base en mecanismos cinéticos propuestos.

\section{MATERIALES Y MÉTODOS}

Se detalla el sistema de reacción, los materiales usados, el análisis del contenido proteico, y la actividad enzimática. Se detalla el método de control de la reacción de hidrólisis proteica y se describe el diseño de experimentos usado. 


\section{Sistema de Reacción}

Se empleó un reactor de vidrio de $1 \mathrm{~L}$ con camisa de circulación de agua para regulación de la temperatura, conectado a un regulador termostático. El control de $\mathrm{pH}$ y registro de temperatura, se hicieron con un electrodo combinado de vidrio LL con diafragma esmerilado fijo (temperatura entre $0-80{ }^{\circ} \mathrm{C}$ ), conectado a un titulador automático Titrando 842 (Metrohm, Suiza), operado por ordenador (software Tiamo 1.2.1). El medio de reacción fue constantemente agitado utilizando un agitador magnético 801 (Metrohm, Suiza)) con velocidad la agitación de $200 \mathrm{rpm}$.

\section{Materiales}

Todos los reactivos usados fueron de grado analítico. El plasma fue comprado a un proveedor comercial, en Medellín-Colombia. Presentaba forma líquida, con $70 \mathrm{~g} / \mathrm{L}$ de proteína. A este se le realizaron controles microbiológicos de mohos y levaduras, coliformes totales y mesófilos, al momento de la recepción. La enzima usada fue Alcalase ${ }^{\circledR} 2,4$ L grado alimenticio (Novozymes, Dinamarca), la cual es una enzima proteolítica producida por fermentación sumergida de una cepa seleccionada de Bacillus licheniformis. El componente principal es Subtilisina A (Subtilisina Carlsberg).

\section{Análisis del contenido proteico}

La concentración de proteínas fue determinada por el método de Bradford, (Bradford, 1976). La curva patrón se construyó empleando Albumina Bovina referencia A7030 de SIGMA- ALDRICH ${ }^{\circledR}$. La absorbancia se midió a temperatura de $20{ }^{\circ} \mathrm{C}$, longitud de onda de $595 \mathrm{~nm}$, empleando un espectrofotómetro UV-1700 Pharma Spec de SHIMADZU ${ }^{\circledR}$.

\section{Actividad enzimática}

Para la determinación de la actividad enzimática del complejo comercial Alcalase ${ }^{\circledR}$ se utilizó el método modificado de Takami et al. (1989). De acuerdo con este procedimiento una alícuota de $0,25 \mathrm{~mL}$ de enzima se incuba con 2,5 mL de solución de caseína 0,6\%, en tampón Glisina: $\mathrm{NaCl}: \mathrm{NaOH}(50 \mathrm{mM}, \mathrm{pH} 8,7$ y temperatura de $\left.60^{\circ} \mathrm{C}\right)$. La reacción de detiene por adición de $2,5 \mathrm{ml}$ de solución de TCA $(0,11 \mathrm{M}$ ácido Tricloroacetico, 0,22M acetato de sodio, 0,33 M ácido acético), la mezcla es centrifugada por $15 \mathrm{~min}$, el sobrenadante se mezcla con $2,5 \mathrm{~mL}$ de $\mathrm{Na}_{2} \mathrm{CO}_{3} 0,5 \mathrm{M}$ y reactivo de Folinciacalteu, por $30 \mathrm{~min}$. La densidad óptica se midió a $660 \mathrm{~nm}$ empleando un espectrofotómetro UV-1700 Pharma Spec de SHIMADZU ${ }^{\circledR}$. Para la determinación se empleó una curva estándar de solución de Tirosina entre 0-1000 mg/L. En este método una unidad enzimática $(U)$ se define como la cantidad de enzima que produce un ug de tirosina en 1 min (Takami et al. 1989).

\section{Control de la reacción de hidrólisis proteica}

Estudios previos de hidrólisis de hemoglobina bovina (Márquez y Vázquez, 1999) y sangre porcina (PérezGálvez R et al., 2011) con Alcalase ${ }^{\circledR} 2,4 \mathrm{~L}$, revelan que concentraciones iníciales de sustratos del orden de 1-10 g/L de proteína, presentan buen comportamiento respecto a la velocidad de formación de péptidos. En este caso se empleó, plasma entero bovino con $6 \mathrm{~g} / \mathrm{L}$ de proteína. Durante la reacción se ajustó el pH (Adicionando $\mathrm{NaOH} 1 \mathrm{~N}$ ), la relación enzima-sustrato $(\mathrm{E} / \mathrm{S})$ y la temperatura en la solución proteica, según lo indica el diseño de experimentos. La reacción que se dejó por dos horas.

La reacción a pH alcalino, se monitoreó para la determinación del grado de hidrólisis (GH), expresado como la relación entre el número de enlaces peptídicos cedidos en la hidrólisis ( $h$ ) y el número de enlaces peptídicos totales en la proteína nativa por unidad de peso $\left(h_{t}\right)$. Para éste caso, se empleó un $h_{t}$ de 8,3 Eqv/Kg (Márquez y Vázquez, 1999). El método empleado para la determinación del grado de hidrólisis es el de valoración del protón o método del pH-estático (ecuaciones 1-3) (Márquez y Vázquez, 1999). Éste consiste en mantener constante el pH del medio de reacción por adición de Hidróxido de sodio $1 \mathrm{~N}$, para neutralizar los protones formados en la disociación del grupo carboxilo terminal, los cuales se reparten de acuerdo al equilibrio de protonización de los grupos $\alpha$-amino liberados (Adler-Nissen, 1986).

$$
\begin{aligned}
& G H=\frac{B N_{B}}{M_{P}} \frac{1}{\alpha} \frac{1}{h_{t}} * 100 \\
& \alpha=\frac{10^{p H-p K}}{1+10^{p H-p K}}
\end{aligned}
$$


$p k=7,8+\frac{298-T}{298 * T} 2400$

En estas ecuaciones, $B$ es el Volumen consumido de base en $L, N_{B}$ es la Normalidad de la base (Eqv/L), Mp es Masa de la proteína en $\mathrm{Kg}$ y a en la ecuación (2) es el grado de disociación de los grupos a- $\mathrm{NH}_{2}$ liberados en la reacción, el cual depende del pK, que a su vez depende de la temperatura, como puede apreciarse en la ecuación (3) (Valencia et al., 2014).

\section{Diseño de Experimentos}

Se planteó un diseño de experimentos Central Compuesto rotable (CCR), con tres factores (Temperatura, $\mathrm{pH}$ y la relación $\mathrm{E} / \mathrm{S}$ ) y cinco niveles, según se indica en la tabla 1. La variable respuesta fue el $\mathrm{GH}$. Se realizó el análisis de varianza (ANOVA) y el ajuste del modelo en la forma de la ecuación (4), utilizando el software Statgraphics Centurion XVI. El ajuste del modelo empírico se verificó por medio del $\mathrm{R}^{2}$ y el $\mathrm{R}^{2}$ Ajustado, según las ecuaciones (5) y (6). La significancia de los coeficientes estimados en el modelo se probó con el estadístico $\mathrm{F}$ y un nivel de confianza de $95 \%$ (Valor $-\mathrm{P}<0,05$ ).

Tabla 1. Variables y niveles empleadas en el diseño CCR.

\begin{tabular}{|c|c|c|c|c|c|c|c|}
\hline \multirow{2}{*}{ Factores } & \multicolumn{3}{|c|}{ Símbolo } & \multicolumn{5}{c|}{ Niveles Codificados } \\
\cline { 2 - 8 } & Codificado & Sin Codificar & $-1,68$ & -1 & 0 & 1 & 1,68 \\
\hline $\mathrm{T}\left({ }^{\circ} \mathrm{C}\right)$ & $\mathrm{X} 1$ & $\mathrm{x} 1$ & 50 & 55 & 62,5 & 70 & 75 \\
\hline $\mathrm{pH}$ & $\mathrm{X} 2$ & $\mathrm{x} 2$ & 7,4 & 7,7 & 8,2 & 8,7 & 9 \\
\hline $\mathrm{E} / \mathrm{S}(\mathrm{w} / \mathrm{w})$ & $\mathrm{X} 3$ & $\mathrm{x} 3$ & 5,0 & 6,0 & 7,5 & 9,0 & 10 \\
\hline
\end{tabular}

$Y_{j}=\alpha_{0} \sum_{i=1}^{3} \alpha_{i} X_{i}+\sum_{i=1}^{3} \alpha_{i i} x_{i}^{2} \sum_{i<j}^{j=3} \alpha_{i j} x_{i j}$

Donde $Y_{j}$ es la respuesta predicha $(\mathrm{GH}), \mathrm{X}_{i}$ y $\mathrm{X}_{j}$ desde $i, j=1$ hasta 3 son las variables independientes 0 factores; $\alpha_{0}, \alpha_{i}, \alpha_{i j}, \alpha_{i j}$, son los términos independientes, lineales, cuadráticos y de interacción del polinomio.

$$
\begin{aligned}
& \mathrm{R}^{2}=\frac{\mathrm{SC}_{\text {total }}-\mathrm{SC}_{\text {error }}}{\mathrm{SC}_{\text {total }}} \times 100 \\
& \mathrm{R}_{\mathrm{AJ}}^{2}=\frac{\mathrm{CM}_{\text {total }}-\mathrm{CM}_{\text {error }}}{\mathrm{CM}_{\text {total }}} \times 100
\end{aligned}
$$

\section{OPTIMIZACIÓN DEL MODELO}

El modelo matemático construido por regresión con los datos codificados de las variables se sometió a un proceso de optimización empleando métodos determinísticos y no determinísticos de análisis, con el fin de determinar las condiciones de T, pH y E/S que maximizan el GH de la reacción de hidrólisis. Los métodos se describen a continuación:

\section{Optimización por MSR}

Mediante un diseño cuidadoso de experimentos se buscó optimizar el GH que está influenciado por algunas variables independientes (variables de entrada). Para su análisis se plantean una serie de experimentos, en los que se realizan cambios en las variables de entrada con el fin de identificar las razones de los cambios en la variable respuesta. Este enfoque empírico es a menudo adecuado para la mejora de procesos en un entorno industrial. Luego de planteado y evaluado el modelo, es posible encontrar las condiciones óptimas de trabajo de las variables de entrada. Una de las herramientas generalmente empleadas para este fin, es optimizar por metodología de superficie de respuesta, la cual se basa en el método de la máxima pendiente en ascenso, que consiste en recorrer la trayectoria de máxima pendiente, que conduce a la dirección del máximo aumento en la respuesta. La región de exploración son los niveles codificados de los factores 
evaluados. Para resolver estos sistemas se emplea el programa de optimización Statgraphics Centurion ${ }^{\circledR}$. Además, los valores óptimos de los factores codificados ( $T, \mathrm{pH}$ y E/S), se emplearon en la ejecución de tres replicas experimentales, cuyos resultados se compararon con los predichos por el modelo polinomial ajustado.

\section{Optimización por AGs}

Los algoritmos genéticos son un conjunto de procedimientos informáticos de búsqueda y optimización basado en el concepto de la selección natural y la genética (Correia et al, 2005). Son métodos robustos de búsqueda, que permiten tratar problemas de optimización donde el objetivo es encontrar un conjunto de datos que minimizan o maximizan una función de adaptación. Estos algoritmos operan sobre un conjunto de individuos, generalmente representado por una cadena binaria comprendida entre 0 y 1 . La rutina de optimización por algoritmos genéticos se resuelve mediante el toolbox de optimización "optimtool" de MATLAB $98^{\circledR}$.

La estructura de funcionamiento del AG, se muestra en la figura 1. El algoritmo parte de una población inicial de $n$ individuos aleatorios, los cuales son cadenas de caracteres que muestran una factible solución al problema que se resuelve. Cuando la población es creada, una función, conocida como la función de aptitud, es encargada de evaluar o calificar cada uno de los individuos, permitiendo que sean seleccionados de este proceso, los individuos que formarán la base de la siguiente generación. Una pequeña parte de la nueva población se generará mediante la eventual mutación de alguno de los individuos seleccionados y el resto de la nueva población se generará mediante el cruzamiento entre pares de individuos conocidos como padres, los cuales compartirán características en un nuevo individuo. Después de terminar el número de generaciones se selecciona el individuo con mejor desempeño, el cual representa la solución óptima.

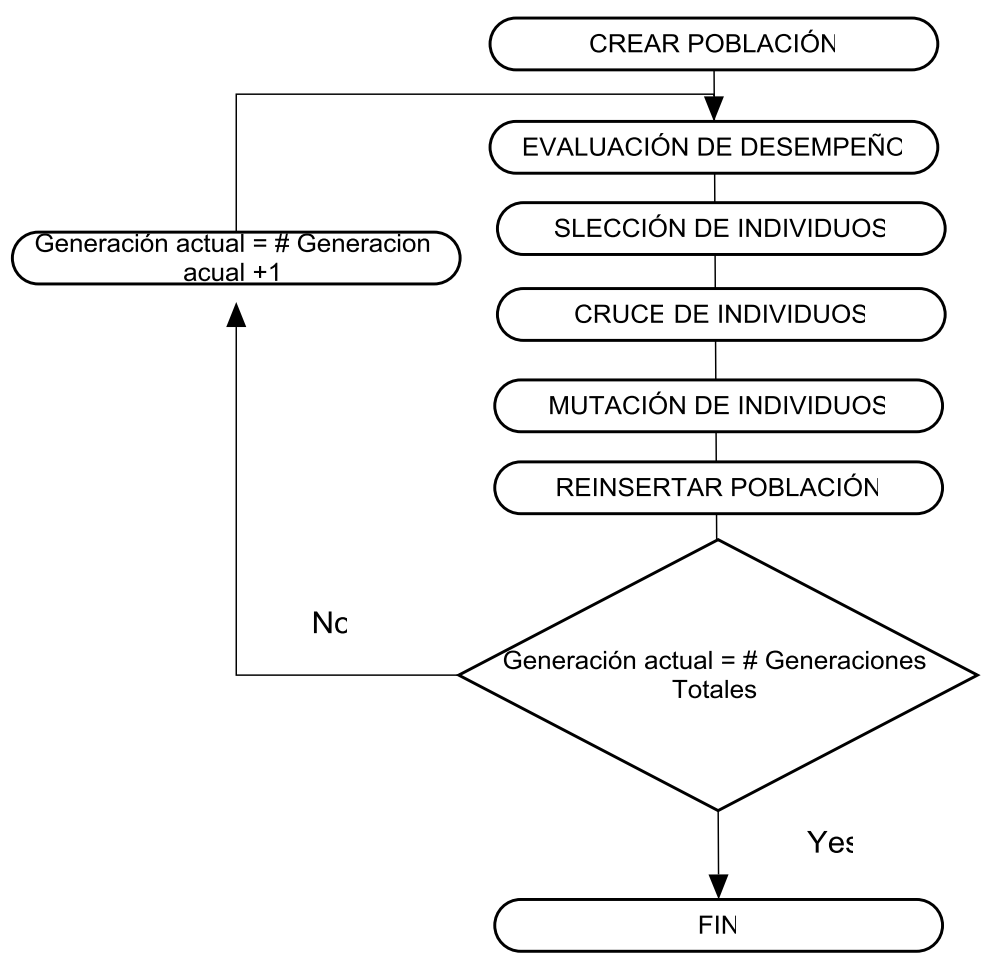

Fig. 1. Estructura básica de funcionamiento del AG.

Los individuos que se someterán a cruzamiento y mutación, pueden ser seleccionados mediante el proceso de selección de Ruleta, empleado por Goldberg, en su algoritmo genético simple (SAG), el cual favorece la selección de los mejores individuos, sin dejar de lado la posibilidad de que se escojan algunos de los peores, permitiendo así la diversidad a la población de soluciones (Correia et al., 2005). Los parámetros de cálculo para el AG se muestran en la tabla 2. Se establecieron teniendo en cuenta que el tamaño de la población, la tasa de cruce y la tasa de mutación son factores importantes en el rendimiento del algoritmo. Por un lado, un gran número de individuos o una mayor tasa de cruce permiten la exploración del espacio de soluciones y reduce la posibilidad de alcanzar una mala solución, pero si estos son demasiado grandes, el resultado es un elevado gasto computacional.

La población inicial se refiere entonces a las posibles soluciones del problema de optimización, donde cada una de estas se conoce como un individuo, por tanto una población de 20 está formada por un conjunto 
inicialmente aleatorio de valores de T, pH y E/S en la región experimental estudiada, indicados obviamente como una cadena binaria. Las etapas de selección, cruce y mutación definen los nuevos valores de las tres variables independientes $(\mathrm{T}, \mathrm{pH}$ y E/S) en el espacio experimental. Estos valores son evaluados en la función de adaptación a través de la determinación de sus respectivos GHs, procedimiento que se repite con los nuevos individuos hasta completar el número de generaciones o hasta donde exista convergencia.

Tabla 2. Parámetros de cálculo para el AG del problema de optimización.

\begin{tabular}{|c|c|}
\hline Población Inicial & 20 \\
\hline Número de generaciones & 60 \\
\hline Tipo de cruce & Uno Solo \\
\hline Función de Mutación & Gaussiana \\
\hline Tasa de cruzamiento & 0,8 \\
\hline Tipo de Selección & Ruleta \\
\hline Variables & 3 \\
\hline Función de evaluación (Max) & Ecuación (8) \\
\hline
\end{tabular}

El proceso de selección se encarga de elegir cuales de los individuos pasarán a las siguientes generaciones, donde un individuo es seleccionado si tiene buen valor de aptitud en la función de evaluación (ecuación 8). Después de la selección se efectúa el cruce, este consta básicamente tres pasos. En primer lugar, dos cadenas de individuos (conocidos como los padres) son seleccionados al azar de las opciones de apareamiento (individuos aptos según el proceso de selección). Luego se selecciona una ubicación arbitraria llamada la zona de cruce (una solo zona para este algoritmo) en ambas cadenas al azar. Por último las cadenas siguientes al sitio de cruce se intercambian entre dos cadenas de padres para formar dos cadenas de descendencia. Este cruce se produce de manera aleatoria y en nuestro caso con una tasa de 0,8 . La mutación por su parte consiste en la alteración aleatoria de cada gen componente de la cadena este y asegura que ningún punto del espacio de análisis tenga cero probabilidades de ser examinado, lo cual asegura la convergencia del algoritmo. El método empleado en este caso, consiste en sumar al valor actual del gen una cantidad elegida aleatoriamente a partir de una distribución Gaussiana N $(0, \sigma)$ y luego, si es necesario, reducir el valor resultante al rango de valores permitidos (D'Ambrosio et al., 2013).

\section{Modelo de inhibición por sustrato}

Estudios previos, sugieren, que existe un efecto sobre el GH de la reacción, que puede ser atribuido a las concentraciones de sustrato, en especial cuando la reacción tiene lugar a concentraciones de sustrato relativamente elevados (Figueroa et al, 2012). Con el objetivo de evaluar el efecto del sustrato, se planteó un nuevo diseño experimental a diferentes concentraciones de sustrato y enzima, el cual se muestra en la tabla 3. La temperatura y el pH, se fijaron de acuerdo con los resultados de la optimización de MRS: T=60,4 ${ }^{\circ} \mathrm{C}$ y el $\mathrm{pH}=9,0$. El tiempo de reacción fue de 2 horas. Los datos fueron tomados por duplicado.

Tabla 3. Experimentos para distintas concentraciones de sustrato y enzima inicial en el sistema Plasma Bovino-Alcalasa 2,4L $\mathrm{pH}=9,0, \mathrm{~T}=60,4^{\circ} \mathrm{C}$.

\begin{tabular}{|c|c|}
\hline $\begin{array}{c}\text { Sustrato Inicial }\left(\mathrm{S}_{0}\right) \\
(\mathrm{g} / \mathrm{L})\end{array}$ & $\mathrm{E}_{0} / \mathrm{S}_{0}(\mathrm{AU} / \mathrm{g})$ \\
\hline \multirow{2}{*}{4} & 0,0735 \\
\cline { 2 - 2 } & 0,1225 \\
\cline { 2 - 2 } & 0,245 \\
\hline \multirow{2}{*}{6} & 0,1225 \\
\hline \multirow{2}{*}{12} & 0,245 \\
\hline
\end{tabular}

Modelo Matemático de Inhibición por sustrato

Se propuso un modelo matemático basado en el mecanismo de acción enzimática (González-Tello et al., 1994), que establece una relación exponencial entre la velocidad de la reacción, la concentración inicial de sustrato $\left(S_{0}\right)$ y de enzima $\left(e_{0}\right)$, con dos parámetros de ajuste $\boldsymbol{a}^{\prime}$ y b' Ecuación (7), que fueron determinados con la función "Isqcurvefit" del "toolbox" de MATLAB $98^{\circledR}$, la cual resuelve problemas de ajuste de datos de curvas no lineales. 
$\frac{d(G H)}{d t}=a^{\prime} \operatorname{EXP}\left[-b^{\prime}(G H)\right]$

De acuerdo con estos resultados es posible obtener los parámetros cinéticos del mecanismo cinético a través de un ejercicio de linealización y análisis de interceptos y pendientes de los gráficos de: $b^{-1} \mathrm{Vs}_{0}$; $a b^{-}$ ${ }^{1} \mathrm{Vs} \mathrm{e}_{0} \mathrm{~S}_{0}^{-1} ; \mathrm{r}^{-1} \mathrm{Vs} \mathrm{S}_{0}^{-1}$ (Lineweaver-Burk para varias concentraciones de sustrato) (Qi Wei, 2006).

\section{RESULTADOS Y DISCUSIÓN}

\section{Ajuste del Modelo}

Los resultados obtenidos tras la ejecución del diseño experimental propuesto para el análisis de la hidrólisis enzimática de plasma entero de bovino con Alcalase ${ }^{\circledR} 2,4 \mathrm{~L}$, se muestran en la tabla 4 . Los experimentos fueron planteados para determinar el tipo de relación de las variables: Temperatura (X1), el pH (X2) y la relación enzima/sustrato (X3), sobre el GH del plasma. Los resultados del análisis de varianza (ANOVA) para la prueba de significancia de los coeficientes del modelo se muestran en la tabla 5 . De acuerdo con este análisis, la temperatura es el factor más significativo en el GH de la reacción, teniendo en cuenta que los términos lineales y cuadráticos muestran un efecto significativo elevado con valores $p \leq 0,05$ y $p \leq 0,01$ respectivamente.

En el rango experimental de trabajo, los efectos cuadráticos y de interacción del pH y E/S no presentan efectos significativos sobre la variable de respuesta con valores $p>0,05$, al igual que las interacciones de la temperatura, por lo que no fueron tenidas en cuenta para la definición del modelo. Por otro lado se evidencia una dependencia lineal del GH con la $E / S$, con probabilidades asociadas $(p=0,0122)$. El valor máximo de este factor se alcanza justo en el mayor nivel evaluado $(E / S=10,0)$. El aumento de $E / S$ eleva la velocidad de hidrólisis como es común que ocurra cuando las concentraciones de enzimas son relativamente bajas en relación con las concentraciones críticas que inducen saturación en sistemas de reacción de hidrólisis enzimática. Estos datos se emplearon en el ajuste de un modelo polinomial de segundo orden, cuyos coeficientes fueron calculados por regresión múltiple.

Tabla 4. Diseño Central Compuesto y GHs Obtenidos

\begin{tabular}{|c|c|c|c|c|}
\hline \multirow[b]{2}{*}{ Exp } & Temperatura (ㅇ C) & $p H$ & $E / S(\% P / V)$ & \multirow[b]{2}{*}{$\mathrm{GH}(\%)$} \\
\hline & $x 1$ & $x 2$ & $x 3$ & \\
\hline 1 & 55 & 7,7 & 6,0 & 14,4 \\
\hline 2 & 55 & 7,7 & 9,0 & 18,3 \\
\hline 3 & 55 & 8,7 & 6,0 & 16,1 \\
\hline 4 & 55 & 8,7 & 9,0 & 20,2 \\
\hline 5 & 62,5 & 8,2 & 7,5 & 19,7 \\
\hline 6 & 62,5 & 8,2 & 5,0 & 18,8 \\
\hline 7 & 62,5 & 8,2 & 10,0 & 21,4 \\
\hline 8 & 62,5 & 9 & 7,5 & 21,1 \\
\hline 9 & 62,5 & 8,2 & 7,5 & 21,5 \\
\hline 10 & 62,5 & 8,2 & 7,5 & 20,4 \\
\hline 11 & 62,5 & 8,2 & 7,5 & 20,2 \\
\hline 12 & 50 & 8,2 & 7,5 & 10,6 \\
\hline 13 & 70 & 7,7 & 9,0 & 14,6 \\
\hline 14 & 70 & 7,7 & 6,0 & 12,8 \\
\hline 15 & 70 & 8,7 & 9,0 & 15,2 \\
\hline 16 & 62,5 & 7,4 & 7,5 & 18,7 \\
\hline 17 & 70 & 8,7 & 6,0 & 13,9 \\
\hline 18 & 75 & 8,2 & 7,5 & 9,7 \\
\hline
\end{tabular}


El modelo es descrito en la ecuación (8), luego del proceso de reducción, donde se despreciaron los términos que no fueron significativos $(p<0.05)$.

$\mathrm{GH}=19,89-1,02 \times 1+0,69 \times 2+1,12 \times 3-3,81 \times 1^{2}$

Tabla 5. Análisis de varianza (ANOVA) para los coeficientes regresión del modelo ajustado.

\begin{tabular}{|c|c|c|c|c|c|}
\hline Fuente & $\begin{array}{c}\text { Suma de } \\
\text { Cuadrados }\end{array}$ & Gl & $\begin{array}{c}\text { Cuadrado } \\
\text { Medio }\end{array}$ & Razón-F & Valor-P \\
\hline Lineal & & & & & \\
\hline Temperatura (T) & 14,3115 & 1 & 14,3115 & 24,55 & 0,0158 \\
\hline $\mathrm{pH}$ & 6,44347 & 1 & 6,44347 & 11,05 & 0,0449 \\
\hline $\mathrm{E} / \mathrm{S}$ & 17,2109 & 1 & 17,2109 & 29,52 & 0,0122 \\
\hline Cuadrático & & & & & \\
\hline $\mathrm{T}^{2}$ & 185,973 & 1 & 185,973 & 318,99 & 0,0004 \\
\hline Falta de ajuste & 14,8542 & 10 & 1,48542 & 2,55 & 0,2388 \\
\hline Error Puro & 1,74901 & 3 & 0,583003 & & \\
\hline Total (corr.) & 240,542 & 17 & & & \\
\hline
\end{tabular}

El análisis de varianza (ANOVA) demuestra que el modelo representa adecuadamente los datos experimentales, teniendo en cuenta que el $R^{2}$ es de 0,931 , lo que indica que éste describe la influencia de las variables independientes sobre el GH de las proteínas del plasma bovino en un porcentaje satisfactorio del 93\% aproximadamente. En la figura 2, están representados los valores estimados en comparación con los datos observados de la variable respuesta, evidenciando la buena correlación, puesto que los puntos cercanos alrededor de la diagonal que indica el óptimo del modelo, muestran que la desviación entre dichos valores fue mínima. Para este modelo el valor del estadístico $R^{2}$ ajustado es de 0,910 , lo que nos dice, que efectivamente los factores estudiados, junto con sus interacciones explican en un porcentaje satisfactorio la variabilidad observada en el $\mathrm{GH}$, luego es muy buena la descripción matemática de la hidrólisis de proteínas de plasma bovino del modelo propuesto en la ecuación (8).

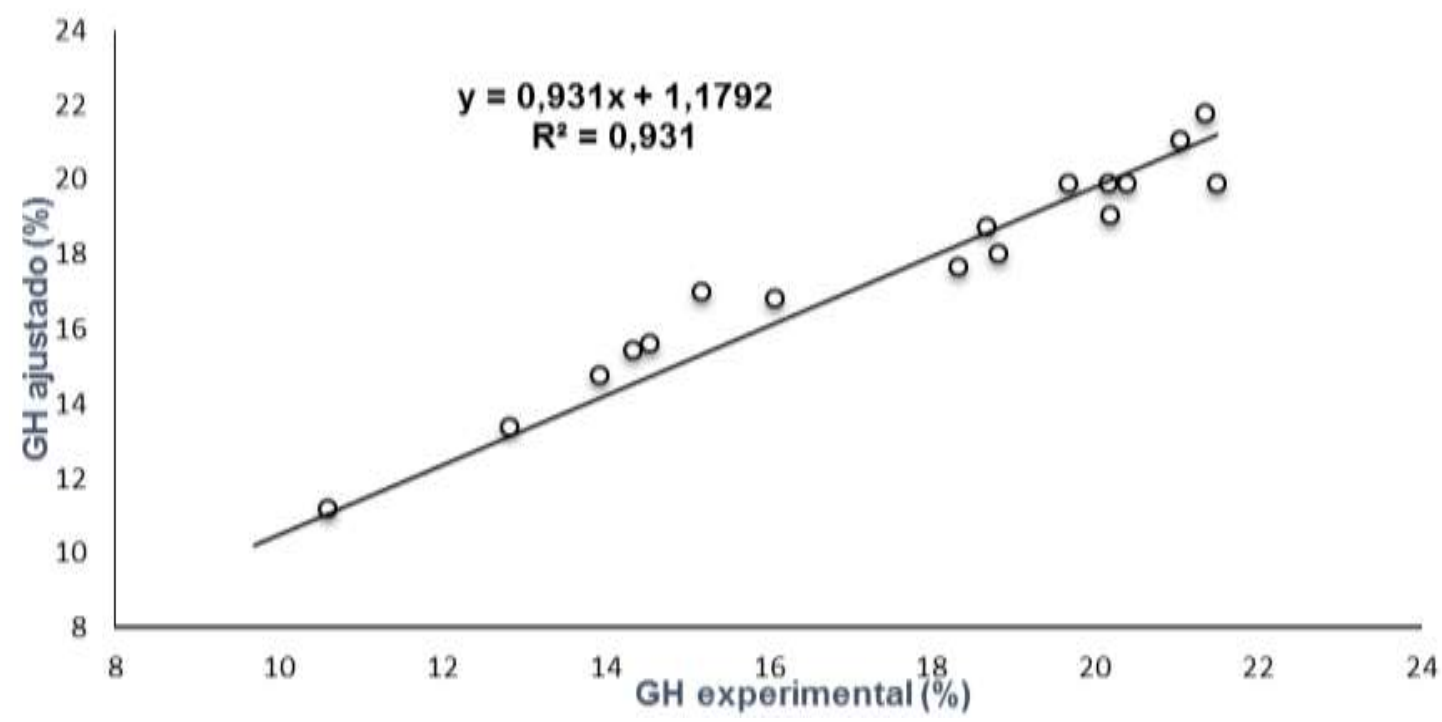

Fig. 2. Grados de hidrólisis experimentales y ajustados por el polinomio de segundo orden.

\section{Optimización de las condiciones de hidrólisis}

En la tabla 6, se muestran los resultados de la optimización empleando MSR y AGs, aquí se registran los valores óptimos de los factores evaluados (codificados y reales) y el GH (variable respuesta) predicho frente al valor experimental obtenido. Se observa que los valores óptimos para el GH, reportados por la MSR y AG, son similares en magnitud, aproximadamente de $23 \%$, con niveles codificados muy similares de las variables de estudio. Esto indica, que para este caso las rutinas de optimización tienen por lo menos la 
misma capacidad de búsqueda, lo cual puede estar asociado a que el polinomio explorado describe un comportamiento relativamente sencillo, en la región de trabajo evaluada.

Para confirmar la validez del modelo matemático propuesto, se llevaron a cabo tres corridas experimentales bajo las condiciones óptimas establecidas. Estos datos muestran una vez más el buen ajuste del modelo y evidencian el éxito de la rutina de maximización del GH de la reacción de hidrólisis empleando ambos métodos. La prueba de falta de ajuste es contundente y corrobora la calidad de ajuste del modelo $(p=$ 0,2388 ). La buena precisión de los experimentos ( $S D \pm 0,17$ ) sugiere que efectivamente los valores encontrados de los niveles para cada factor evaluado son condiciones óptimas de trabajo para este sistema de reacción. En la Figura 3 se muestra el comportamiento gráfico de $\mathrm{GH}$ en función del pH y $\mathrm{T}$, definida por la ecuación 8, con una relación E/S de $7.5 \%$. Aquí se observa un aumento del GH con el aumento de la temperatura hasta regiones cercanas a la mitad del intervalo, después del cual presenta un descenso. En teoría, un incremento en la temperatura aumenta la energía cinética a las moléculas, pero debido a la naturaleza proteica de las enzimas su estructura terciaria se ve comprometida con las elevadas temperaturas lo que conduce a la pérdida de actividad catalítica (Pérez-Gálvez R et al., 2011).

Los óptimos de temperatura y $\mathrm{pH}$, concuerdan con los rangos de actividad de la Alcalase ${ }^{\circledR} 2,4 \mathrm{~L}$. Considerando la naturaleza bioquímica de la reacción, aumentos importantes se registran en el rendimiento de la misma cuando se suscitan leves incrementos en la temperatura hasta ciertos niveles indicados, esto contrasta con las disminuciones observadas en la velocidad de ruptura de enlaces peptídicos cuando la temperatura supera un valor límite que provee desnaturalización y perdida de la actividad catalítica de enzimática (Prieto, 2008).

Fenómeno similar ocurre con el $\mathrm{pH}$, puesto que es sabido que cambios en el $\mathrm{pH}$, pueden influenciar la disociación de grupos activos de la enzima, afectando la dinámica de asociación de esta con el sustrato (Shi, 2005). Los efectos de la solubilización de proteínas son positivos en la medida en que el peso molecular medio de los polipéptidos contenidos en la reacción de hidrólisis es cada vez menor (PérezGálvez et al., 2011), para las 2 horas de reacción, cuando se ha alcanzado un GH cercano a los $23 \%$, las fracciones cercanas a los $10 \mathrm{kDa}$, han disminuido enormemente, por lo que se cree, a juzgar por los resultados expuestos, que la proporción mayoritaria para GH es de un orden menor a los $5 \mathrm{kDa}$.

Tabla 6. Condiciones óptimas de los factores experimentales en la hidrólisis enzimática de proteínas de plasma Bovino.

\begin{tabular}{|c|c|c|c|c|c|c|c|}
\hline \multicolumn{5}{|c|}{ Condiciones Optimas } & \multicolumn{3}{|c|}{$\mathrm{GH}(\%)$} \\
\hline Variables & $\begin{array}{c}\text { Valor } \\
\text { Codificado } \\
\text { con MSR }\end{array}$ & $\begin{array}{l}\text { Valor sin } \\
\text { Codificar } \\
\text { con MSR }\end{array}$ & $\begin{array}{c}\text { Valor } \\
\text { codificado } \\
\text { con } A G\end{array}$ & $\begin{array}{l}\text { Valor sin } \\
\text { Codificar } \\
\text { con } A G\end{array}$ & $\begin{array}{l}\text { Simulado } \\
\text { con MSR }\end{array}$ & $\begin{array}{c}\text { Simulado } \\
\text { con } A G\end{array}$ & Experimental \\
\hline $\mathrm{T}\left({ }^{\circ} \mathrm{C}\right)$ & $-0,139$ & 61,5 & $-0,141$ & 61,4 & \multirow{3}{*}{22,999} & \multirow{3}{*}{23,002} & \multirow{3}{*}{$22,91 \pm 0,17$} \\
\hline $\mathrm{pH}$ & 1,682 & 9,0 & 1,680 & 9,0 & & & \\
\hline$E / S(w / w)$ & 1,679 & 10,0 & 1,682 & 10,0 & & & \\
\hline
\end{tabular}

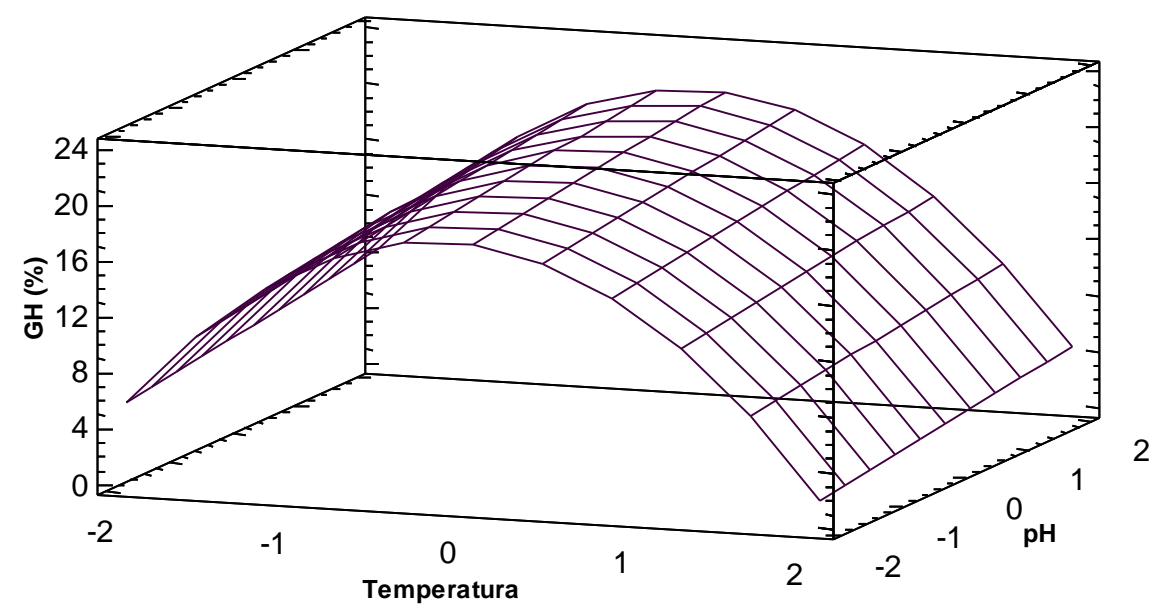

Fig. 3. Superficie de respuesta para el modelo ajustado $E_{0} / S_{0}=7,5(\% P / V)$. 


\section{Influencia de la concentración de sustrato}

En la figura 4, se muestran los resultados experimentales para la hidrólisis enzimática del sustrato en estudio. Esto indica, que efectivamente para concentraciones de sustratos inicial del orden de $12 \mathrm{~g} / \mathrm{L}$, el rendimiento de la reacción se ve considerablemente comprometido. Aquí, se puede ver claramente, que la velocidad de hidrólisis (dGH/dt) disminuye enormemente en el estado medio y final de la reacción. Investigaciones previas (Márquez y Vázquez, 1999; Qi y Hi, 2006; Figueroa et al., 2012) indican que la disminución en la tasa de hidrólisis de la reacción responde generalmente a tres factores: (a) disminución en la concentración de enlaces peptídicos susceptibles a la hidrólisis por las proteasas, (b) posible inhibición de las enzimas causada por el sustrato de hidrólisis; (c) desnaturalización térmica de la enzima.

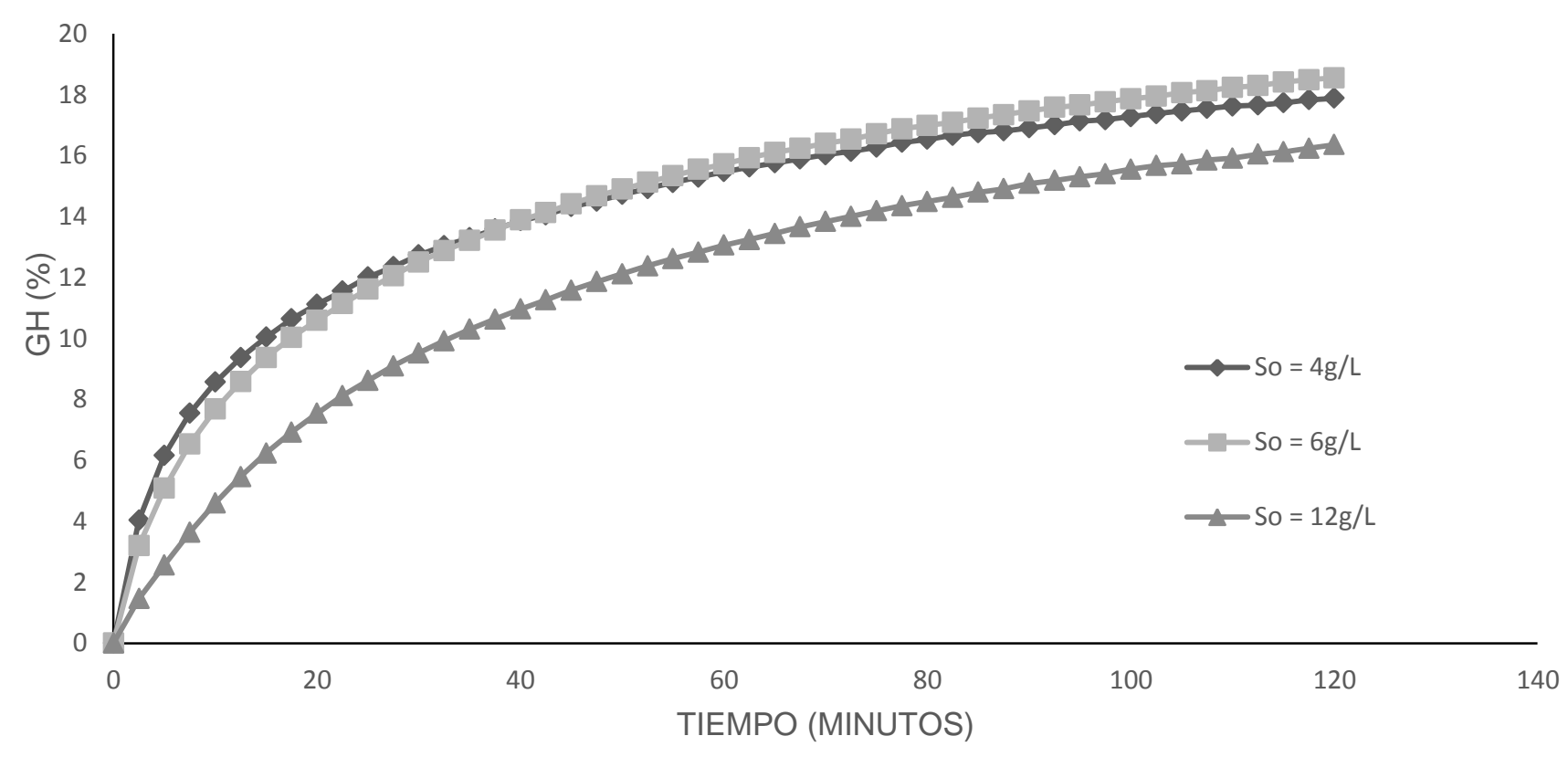

Fig. 4. Comportamiento del $\mathrm{GH}$ con el tiempo, a $\mathrm{pH}=9,0, \mathrm{~T}=61,4^{\circ} \mathrm{C}, \mathrm{E}_{0} / \mathrm{S}_{0}=0,1252 \mathrm{AU} / \mathrm{g}$ y $\mathrm{S}_{0}$ variable.

Analizando las figuras 4, para todos los niveles de enzima y sustrato empleados, se observa la tendencia del grado de hidrólisis hacia valores límites distintos, por lo que es claro que el factor controlante en la velocidad, no es la disminución de enlaces peptídicos disponibles. Por otro lado, según estudios previos, el fenómeno de desactivación enzimática demostró ser influyente en la disminución de la tasa de hidrólisis (Figueroa et al., 2012). Estudios realizados por otros autores, sugieren que un modelo de la forma de la ecuación (7), puede explicarse por medio de una hidrólisis enzimática de orden cero, simultánea con una desnaturalización de la enzima de segundo orden, como lo muestra el siguiente mecanismo:

Suponiendo inactivación por acción del sustrato, tenemos:

$$
\begin{aligned}
& E+\underset{k_{-1}}{\stackrel{k_{1}}{\rightleftarrows} E S} \stackrel{k_{2}}{\rightarrow} E+P \\
& E+E S \stackrel{k_{s}}{\leftrightarrow} S E S \\
& E+E S \stackrel{k_{3}}{\rightarrow} E_{a}+E_{i}+P
\end{aligned}
$$

Donde E, S y P, representan la concentración de enzima, sustrato y producto, respectivamente. ES; es el complejo enzima sustrato, $E_{a}$ y $E_{i}$ indican la concentración de enzima activa e inactiva en la reacción; $k_{s}$ es la constante de inactivación por sustrato, $\mathrm{k}_{2}$ es la constante cinética de velocidad de formación de productos, $\mathrm{k}_{3}$ es la constante de desactivación enzimática.

Según la aproximación de Briggs-Haldane (Rami Tzafriri y Edelman, 2007), la constante de M-M $K_{m}$ es:

$\mathrm{K}_{\mathrm{m}}=\frac{\mathrm{K}_{-}+\mathrm{K}_{2}}{\mathrm{~K}_{1}}$ 
Luego por la aproximación del estado estacionario para el complejo [SES]:

$|S E S|=\frac{|E S||S|}{k_{S}}=\frac{[S]^{2}[E]}{k_{s} k_{m}}$

El balance de enzima en el sistema queda:

$e=E+E S+S E S$

Realizando las sustituciones y suponiendo $[\mathrm{S}]=\mathrm{S}_{0}{ }^{*} \mathrm{~h}_{\text {tot }}$, que está definida como la concentración de enlaces peptídicos iniciales. La expresión final de velocidad en la forma del modelo exponencial es:

$r=s_{0} h_{t o t} \frac{d(\mathrm{GH})}{d t}=a^{\prime} \operatorname{EXP}\left[-b^{\prime}(\mathrm{GH})\right]$

Donde $\boldsymbol{a}^{\prime}$ y $\boldsymbol{b}$ ' son:

$a^{\prime}=\frac{k_{2} k_{s}}{\left(k_{s}+S_{0}\right)} \frac{e_{0}}{s_{0}}$

$b^{\prime}=\frac{k_{3} k_{s} k_{m}}{k_{2}\left(k_{s}+S_{0}\right)}$

Los valores de a' y b' para las diferentes concentraciones de sustrato y enzima, fueron determinados y a través del ajuste con los datos experimentales. En la tabla 7 se resumen estos valores.

En la tabla 8 se muestran los parámetros cinéticos del modelo, evaluados de acuerdo con el proceso de linealización. Los datos obtenidos en los ensayos experimentales se ajustaron a un modelo general de la forma de la ecuación (7). El ajuste del modelo con los datos experimentales fue satisfactorio y los parámetros a' y b' estimados, están de acuerdo con el mecanismo propuesto. Reemplazando el valor de las constantes cinéticas en las ecuaciones (13) y (14), y reordenando la ecuación 12, el modelo puede ser expresado como lo muestra la ecuación (15).

$r=s_{0} h_{t o t} \frac{d(G H)}{d t}=\frac{0,025}{\left(0,0816+S_{0}\right)} \frac{e_{0}^{\prime}}{s_{0}} \operatorname{EXP}\left[-\frac{0,009}{0,3115\left(0,0816+S_{0}\right)}(G H)\right]$

La ecuación diferencial, con estos valores de las constantes cinéticas, se resolvió empleando métodos numéricos (ode15s), a diferentes condiciones de trabajo (E/S variable). Los resultados del ajuste contra los datos experimentales se muestran en la figura 5.

La línea continua representa los valores predichos por el modelo de inhibición por sustrato y los marcadores representan los datos experimentales a $\mathrm{pH}=9,0, \mathrm{~T}=61,4^{\circ} \mathrm{C}$ y $2 \mathrm{~h}$ de reacción.

Tabla 7. Valores de a' y b' para distintos $\left[\mathrm{S}_{0}\right]$ y $\left[\mathrm{e}_{0}\right] /\left[\mathrm{S}_{0}\right], \mathrm{pH}=9,0$ y $\mathrm{T}=61,4^{\circ} \mathrm{C}$

\begin{tabular}{|c|c|c|c|}
\hline \multirow{2}{*}[\mathrm{So}]{$(g / L)$} & $\begin{array}{c}{[\mathrm{EO} / \mathrm{So}]} \\
(\mathrm{AU} / \mathrm{g})\end{array}$ & $a^{\prime}$ & $b^{\prime}$ \\
\hline \multirow{3}{*}{4} & 0,0735 & 1,2673 & 0,2481 \\
\cline { 2 - 4 } & 0,1225 & 3,0605 & 0,2509 \\
\cline { 2 - 4 } & 0,2450 & 7,3919 & 0,2610 \\
\hline \multirow{2}{*}{6} & 0,1225 & 1,9650 & 0,2079 \\
\cline { 2 - 4 } & 0,2450 & 6,5460 & 0,2269 \\
\hline 12 & 0,0980 & 0,5973 & 0,1600 \\
\hline
\end{tabular}


Tabla 8. Parámetros cinéticos del modelo de inactivación por sustrato para el sistema Plasma Bovino-Alcalase ${ }^{\circledR} 2,4 \mathrm{~L}, \mathrm{pH}=9,0$ y $\mathrm{T}=61,4{ }^{\circ} \mathrm{C}$.

\begin{tabular}{|c|c|c|c|}
\hline $\mathrm{k}_{\mathrm{m}}(\mathrm{mol} / \mathrm{L})$ & $\mathrm{k}_{\mathrm{s}}(\mathrm{mol} / \mathrm{L})$ & $\mathrm{k}_{2}(1 / \mathrm{min})$ & $\mathrm{k}_{\mathrm{d}}=\mathrm{k}_{3} * \mathrm{k}_{\mathrm{m}}(1 / \mathrm{min})$ \\
\hline 0,0430 & 0,0816 & 0,3115 & 0,1102 \\
\hline
\end{tabular}

Tal como se puede apreciar en la figura 5, el modelo presenta un buen ajuste con los datos experimentales, con un error relativo promedio con respecto a los datos experimentales de $9,5 \%$, lo cual sugiere que efectivamente, cuando las concentraciones de sustrato y enzima, se trabajan en los rangos evaluados por éste modelo ( $E_{0}$ de $2-10 \%$ y $S_{0}$ de 4 a $\left.12 \mathrm{~g} / \mathrm{L}\right)$, el mecanismo está representado por una cinética de orden cero con respecto al sustrato, simultánea con una desactivación enzimática de segundo orden e inhibición por el sustrato de hidrólisis. Qi y He, (2006), ajustaron un modelo de esta forma para un sistema BSATrypsina, en rangos de concentraciones de sustrato bastante inferiores $(0,25-2,4 \mathrm{~g} / \mathrm{L})$ comparada con las estudiadas en este trabajo.

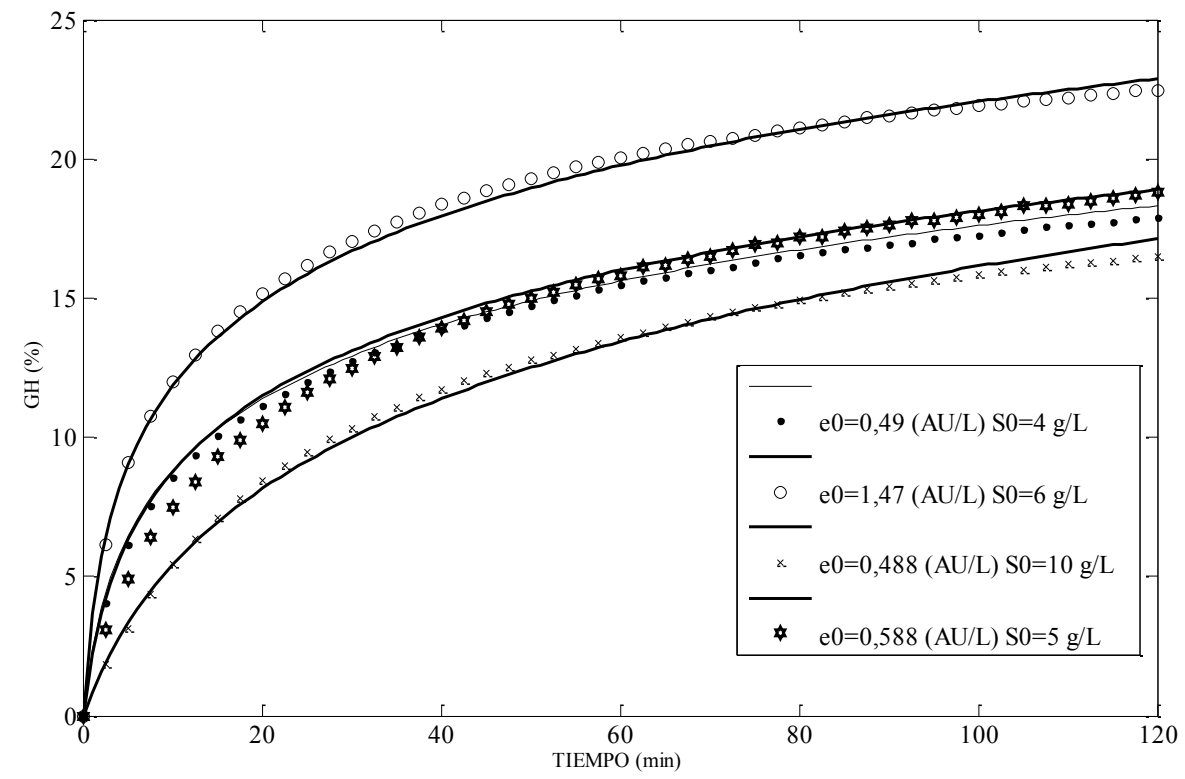

Fig. 5. Comportamiento del GH en función del tiempo a diferentes $\mathrm{e}_{0}$ y $\mathrm{S}_{0}$.

\section{CONCLUSIONES}

Bajo las condiciones del presente estudio se puede afirmar que la reacción de hidrólisis de proteínas de plasma bovino con Alcalase ${ }^{\circledR} 2,4 \mathrm{~L}$ sigue un mecanismo de reacción que representa una cinética de orden cero con respecto al sustrato, simultánea con una desactivación enzimática de segundo orden e inhibición por efectos del sustrato de hidrólisis.

El comportamiento de la reacción puede describirse bien empleando métodos determinísticos como la MSR o métodos probabilísticos como los AG.

Ambos métodos de modelamiento predicen valores similares de los factores que maximizan el GH de la reacción de hidrólisis de proteínas de plasma bovino, como son: Temperatura de $61,4^{\circ} \mathrm{C}, \mathrm{pH}$ de 9,0 y E/S de $10 \%(p / p)$.

\section{REFERENCIAS}

Adler-Nissen, J., Enzymatic hydrolysis of food proteins, 116-124. Elsevier Appl. Sci. Publ., New York, EE.UU (1986)

Arantes G. M., A computational perspective on enzymatic catalysis, doi: 10.1590/S010040422008000200034, Química Nova, (en línea), 31(2), 377-83 (2008)

Benítez R., A Ibarz. y J Pagan., Hidrolizados de proteína: procesos y aplicaciones, Acta Bioquímica Clínica Latinoam: 42(2), 227-36 (2008) 
Bradford M., A rapid and sensitive method for the quantitation of microgram quantities of protein utilizing the principle of protein-dye binding, doi: 10.1016/0003-2697(76)90527-3, Anal Biochem, (en línea), 72(1), 24854 (1976)

Correia D. S. y otros tres autores, Comparison between genetic algorithms and response surface methodology in GMAW welding optimization, doi: 10.1016/j.jmatprotec.2004.04.243, J Mater Process Technol, (en línea), 160(1), 70-6 (2005)

Di Bernardini R. y otros seis autores, Antioxidant and antimicrobial peptidic hydrolysates from muscle protein sources and by-products, doi: 10.1016/j.foodchem.2010.07.004, Food Chem, (en línea), 124(4), 1296-307 (2011)

D'Ambrosio, D. y otros tres autores, 2.7 Genetic Algorithms, Optimization, and Evolutionary Modeling In Treatise on Geomorphology, 74-97, John F Shroder, San Diego, EE.UU (2013)

Eslahi N., F Dadashian. y N. H Nejad., Optimization of enzymatic hydrolysis of wool fibers for nanoparticles production using response surface methodology, doi: 10.1016/j.apt.2012.09.004, Adv Powder Technol, (en línea), 24(1), 416-26 (2013)

Figueroa O. A., J. E. Zapata y G. A. Gutiérrez, Modeling of the kinetics of enzymatic hydrolysis of bovine plasma proteinss, Rev EIA, 17, 71-84 (2012)

Gonzàlez-Tello P. y otros cuatro autores, Enzymatic Hydrolysis of Whey Proteins: I. Kinetic Models, doi: 10.1002/bit.260440415, Biotechnology and Bioengineering, 44 (4), 523-28 (1994)

Liu Q. y otros tres autores, Antioxidant activity and functional properties of porcine plasma protein hydrolysate as influenced by the degree of hydrolysis, doi: 10.1016/j.foodchem.2009.05.013, Food Chem, (en línea), 118(2), 403-10 (2010)

Liu, Jing, G Xiao y Z Daqi., Optimization of the Enzymatic Pretreatment in Oat Bran Protein Extraction by Particle Swarm Optimization Algorithms for Response Surface Modeling, doi: 10.1016/j.Iwt.2008.02.013, LWT - Food Science and Technology (en línea), 41 (10), 1913-1918 (2008)

Márquez M. C. y M. A Vázquez., Modeling of enzymatic protein hydrolysis. doi: 10.1016/S00329592(99)00041-2, Process Biochem, (en línea), 35(1-2), 111-7 (1999)

Möller N. P.y otros tres autores, Bioactive peptides and proteins from foods: indication for health effects, doi: 10.1007/s00394-008-0710-2, Eur J Nutr, (en línea), 47(4), 171-82 (2008)

Muffler K. y otros tres autores, Optimisation of halogenase enzyme activity by application of a genetic algorithm, doi: 10.1016/j.jbiotec.2006.07.008, J Biotechnol, (en línea), 127(3), 425-33 (2007)

Patel P. S., Multi-Objective Job Scheduler using Genetic Algorithm in Grid Computing, International Journal of Computer Applications, 94(14), 34 (2014)

Nedjar-Arroume N. y otros siete autores, Isolation and characterization of four antibacterial peptides from bovine hemoglobin, doi: 10.1016/j.peptides.2006.03.033, Peptides, (en línea), 27(9), 2082-9 (2006)

Park K. J. y C. K Hyun., Antigenotoxic effects of the peptides derived from bovine blood plasma proteins, doi: 10.1016/S0141-0229(02)00024-8, Enzyme Microb Technol, (en línea), 30(5), 633-8 (2002)

Pérez-Gálvez R. y otros cuatro autores, Bi-objective optimisation of the enzymatic hydrolysis of porcine blood protein, doi: 10.1016/j.bej.2010.12.004, Biochem Eng J., (en línea), 53(3), 305-10 (2011)

Prieto C. A., E. M Guadix y A Guadix., Influence of temperature on protein hydrolysis in a cyclic batch enzyme membrane reactor, Biochem Eng J., (en línea), 42(3), 217-23 (2008)

Qi B. y otros cuatro autores, Optimization of Enzymatic Hydrolysis of Wheat Straw Pretreated by Alkaline Peroxide Using Response Surface Methodology, doi: 10.1021/ie8016863, Ind Eng Chem Res, (en línea), 48(15), 7346-53 (2009)

Qi W. y Z.He, Enzymatic hydrolysis of protein: Mechanism and kinetic model, doi: 10.1007/s11458-0060026-9, Front Chem China, (en línea), 1(3), 308-14 (2006) 
Rami Tzafriri A. y E. R. Edelman, Quasi-steady-state kinetics at enzyme and substrate concentrations in excess of the Michaelis-Menten constant, doi: 10.1016/j.jtbi.2006.12.005, J Theor Biol, (en línea), 245(4), 737-48 (2007)

Rubio F. C. y otros cuatro autores. Hidrólisis de caseína con alcalasa, Rev Esp Cienc Tecnol Aliment, 33(1), 59-70 (1993)

Sarkar D. y M. J. Modak, Optimisation of fed-batch bioreactors using genetic algorithms, doi: 10.1016/S0009-2509(03)00095-2, Chem Eng Sci, (en línea), 58(11), 2283-96 (2003)

Seo H. W., y otros cinco autores, Optimization of hydrolysis conditions for bovine plasma protein using response surface methodology, doi: 10.1016/j.foodchem.2015.03.133, Food Chem, (en línea), 185, 106-11 (2015)

Shi D., Z. He y W. Qi, Lumping kinetic study on the process of tryptic hydrolysis of bovine serum albumin, doi: 10.1016/j.procbio.2004.07.009, Process Biochem, (en línea), 40(5), 1943-9 (2005)

Takami H., T. Akiba y K. Horikoshi, Production of extremely thermostable alkaline protease from Bacillus sp. no. AH-101, Appl Microbiol Biotechnol, 30(2), 120-4 (1989) 